\title{
Restoration of Color Images using Image Integration based on SURF Features
}

\author{
M. Sirisha \\ M.Tech (DECS) \\ Department of ECE \\ Vishnu institute of Technology
}

\author{
G. Prasanna Kumar \\ Assistant Professor \\ Department of ECE \\ Vishnu institute of Technology
}

\author{
P. S. N. Murthy \\ Professor \\ Department of ECE \\ Vishnu institute of Technology
}

\begin{abstract}
A flash and long-exposure image pair captured in a dark environment is blurred and noisy. To remove this blur or noise from the image pair there are so many deblurring techniques existing. In this paper implemented a new technique for Restoration of Color Images is introduced. In previous methods, image integration is performed only for well-aligned images, which is a difficult process. This problem can be solved by transferring the color of the flash image using a small fraction of the corresponding pixels in the long-exposure image. Proposed method integrates the color of the long-exposure image with the detail of the flash image using Speeded-Up Robust Features (SURF). This method does not require perfect alignment between the images than the previous methods. Proposed method generates integrated image which has a high contrast than the previous method which is based on SIFT.
\end{abstract}

\section{Keywords}

Blur, integration, SURF, aligned images

\section{INTRODUCTION}

To capture an image in low lighting conditions, the amount of light is not sufficient for recording a clear image. To overcome this problem, some photographic techniques are used. The brightness of the image can be increased by reducing the shutter speed but it causes motion blur in the image. Motion blur occurs due to camera shake or object motion. Motion blur is reduced by decreasing the exposure time but it amplifies noise. De-noising and de-blurring are the major image restoration problems which are studied in the past [1]-[8]. Sharp image is restored from blurred or a very noisy image is a challenging task. Many methods tried to improve image quality with the help of an additional image called flash image [9]-[14]. These methods [9], [10] and [1216] integrate the contrast of a flash image and colorfulness of a no-flash image. In the presence of dim light conditions, these methods have good de-noising or de-blurring capabilities. These methods will not work for misaligned images. These methods work properly if the scene to be captured is stationary.

A new image integration method is proposed for a misaligned image pair called flash image and long-exposure image. To restore an image under dim light conditions, two kinds of images are used. One is Long-exposure image and the other is Flash image. Long exposure image is taken at a slow shutter speed and low ISO sensitivity but it is a blurred image due to low lighting illumination. Flash image is taken with a flash light and at faster shutter speed which has sharp contrast and unnatural color. Proposed method combine the features of both the images to obtain a sharp image in low lighting environment and also this method does not require perfect alignment between the images.

\subsection{Restoring an image captured under low light environment using Image Integration}

In Image processing obtaining a high quality from a blurred or noisy image is one of the most challenging problems [4], [17], [18]. In "Single image blind de-convolution", the sharp image is estimated from the noisy blurred image. To get original image, first kernel is estimated and then non blind deconvolution algorithm is applied. For estimating kernel using multiple images, an old approach called "multi-image blind de-convolution". In this approach the sharp image and blurring kernels are estimated with two or more input images.

\subsection{Various algorithms for the integration of misaligned image pair:}

Integration of a misaligned image pair is performed using correspondence algorithms. Speeded-Up Robust Features (SURF) is a classical approach expressing local features in the images using feature vectors. SURF is a local feature detector and descriptor. It can be used to performing various tasks such as object recognition, image registration, classification or 3D reconstruction. SURF is several times faster than SIFT. SURF is not invariant to image rotation.

\section{RESTORATION OF COLOR IMAGES USING IMAGE INTEGRATION BASED ON SURF FEATURES}

Flash and long-exposure image is an image pair which is taken as input for our algorithm. One is flash image $\mathrm{R}$ and the other is long-exposure image S. Flash image is taken with a flash light, with a low ISO sensitivity and a short exposure time, has a high Signal-to-noise ratio and sharp contrast but contains unnatural color caused by flash light. Long-exposure image is taken at a slower shutter speed but the image is blurred and also contains natural color.

High quality image $\mathrm{Y}$ is reconstructed from image set $\mathrm{R}$ and $\mathrm{S}$ by combining their features.

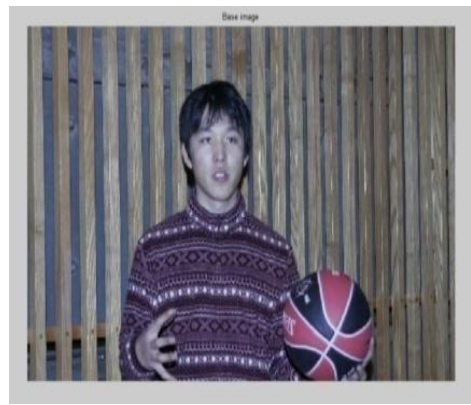

Fig1: Flash image 


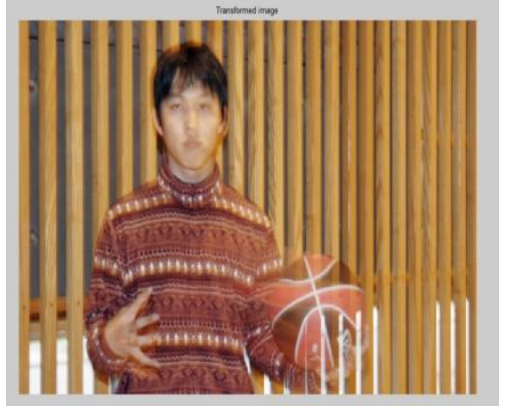

Fig2: Long-exposure image

Find the similarities between the two images, using pixel matching. This method uses only a few set of matched pixel pairs. Color of the flash image depends on the matched pixel pairs.

Our algorithm is explained as follows:

\section{Finding the similarity between two images}

The similarities between the two images are determined first. Long-exposure image is transformed to aligned image set $\mathrm{S}^{\prime}$. SURF is used.

\section{Pixel Selection}

From the aligned image pair, only a fraction of matched pixels are selected.

\section{Image Integration}

The two images are integrated to obtain a high quality image.

\section{Finding the similarity between two images:}

The similarities between the two images flash and long exposure images are determined. There are various algorithms such as patch-based and feature-based methods to find similarity between the two images. Feature-based methods are better than the patch-based method because two images considered here contain different illuminations. SURF is used to detect the similarities between the images.

SURF is the dense correspondence algorithm, which is more reliable than a pixel-based matching.

Long-exposure image is aligned with respect to flash image is expressed as

$$
S^{\prime}:=T_{r}(S)
$$

$\mathrm{S}$ is the long-exposure image

$\mathrm{S}^{\prime}$ is the aligned image

\section{$T_{r}($.$) is the SURF flow operator$}

\section{Pixel Selection in Aligned Image:}

In pixel selection, there are many mismatches and unwanted regions. These unwanted regions are removed to improve the performance of image integration pair. The selection of reliable color correspondence in the set $\mathrm{T}^{\prime}$ is obtained by calculating the difference in local variance between the flash image $\mathrm{R}$ and the aligned long-exposure image $\mathrm{S}^{\prime}$

$$
\boldsymbol{d}_{\boldsymbol{i}}:=\left|\sigma_{r^{z}, i}^{2}-\sigma_{s^{z}, i}^{2}\right| \forall i=1 \text { to } M
$$

Where $\sigma_{r^{z}, i}^{2}:=\sum_{k \in M_{1}(i)}\left(R_{k}^{z}-\bar{R}_{i}^{z}\right)^{2} /\left|M_{1}\right|$ is the local variance.

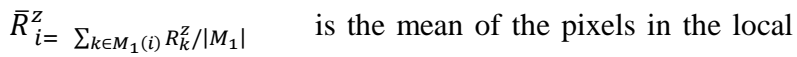
window centered on i. $r_{i}$ and $s_{i}^{\prime}$ are the i-th pixels of R and $S^{\prime}$.
The superscript $\mathrm{z}$ indicates the gray-scale version of the image.

The number of pixels in the window $M_{1(i)}$ is fixed for all $\mathrm{i}$, which is denoted by $\left|M_{1}\right|$

$$
T:=\left\{t \in T^{\prime} \mid d_{t} \leq \tau\right\}
$$

\section{Image Integration}

Flash and long-exposure image pair is taken. Long-exposure image is aligned with respect to flash image by using SURF transform. The two images are integrated by matching the histogram of the flash image with the histogram of aligned long exposure image after removing unreliable pixels.

\section{ALGORITHM IMPLEMENTATION}

The proposed algorithm is based on three main blocks

1. Image registration

In this block, two images flash image and long exposure image are registered.

2. Pixel selection

3. Image integration

The two images flash and aligned long exposure image are integrated in order to obtain high quality image.

\section{Algorithm}

Step1: Take two images R: Flash image, S: long-exposure image

\section{Step2: IMAGE REGISTRATION(R,S)}

$$
S^{\prime}:=T_{r}(\boldsymbol{S})
$$

Define a set that consists of the pixels in the aligned region: $\mathrm{T}^{\prime}$

Step3: PIXEL SELECTION(R, S', T')

$$
\boldsymbol{d}_{\boldsymbol{i}}:=\left|\sigma_{r^{z}, i}^{2}-\sigma_{s^{z}, i}^{2}\right| \forall i=1 \text { to } M
$$

Step4: Selection of reliable pixels

$$
T:=\left\{t \in T^{\prime} \mid d_{t} \leq \tau\right\}
$$

\section{Step5: IMAGE INTEGRATION(R, S', T)}

In this step, resultant image is obtained using flash image $R$, aligned long exposure image $\mathrm{S}^{\prime}$ and reliable pixels in the set $\mathrm{T}$.

\section{EXPERIMENTAL RESULTS}

In this paper, flash and long-exposure images taken in a dark environment as input. Long-exposure image is aligned with respect to Flash image. Flash and long exposure image pair is integrated to obtain high quality image. The obtained high quality image contains color information of long exposure image and details of flash image.

Fig1 and fig2 shows the flash image and long exposure image. The matched SURF points shown in fig3 are obtained by comparing long exposure image with flash image using SURF. Fig4 shows matched inlier points and fig5 shows the recovered aligned image. Fig5 shows the enhanced image which is obtained by calculating reliable pixels. 

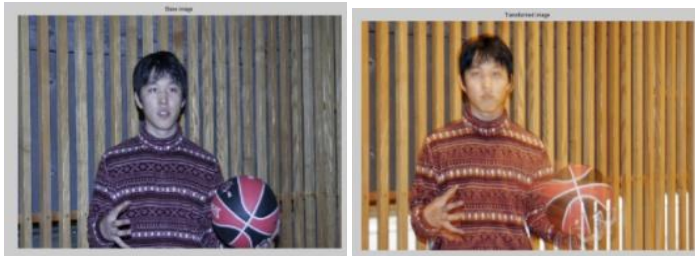

Fig 1: Flash image

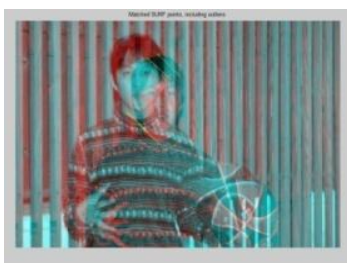

Fig 3: Matched SURF points

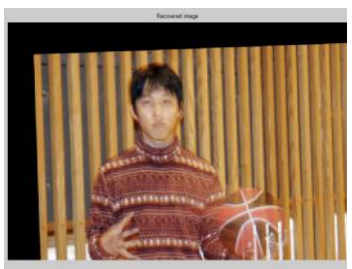

Fig 5: Recovered image ball

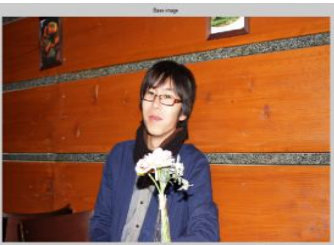

Fig 7: Boy flash image

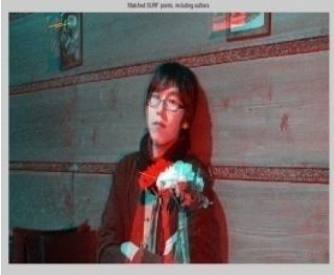

Fig 9: Matched SURF points boy

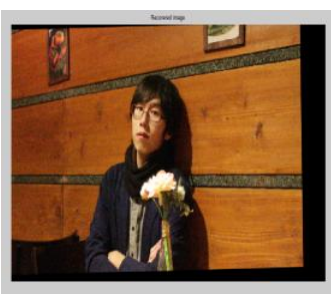

Fig 11: Recovered image boy
Fig 2: Long exposure image

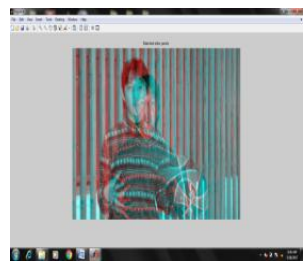

Fig 4: Matched inlier points

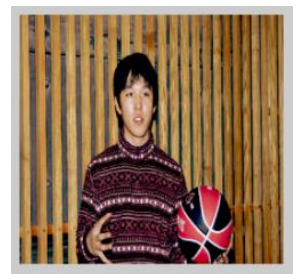

Fig 6: Enhanced image ball

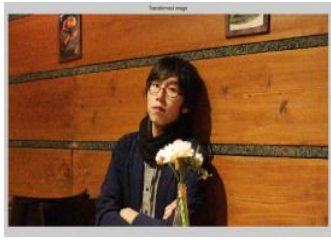

Fig 8: Boy long exposure image

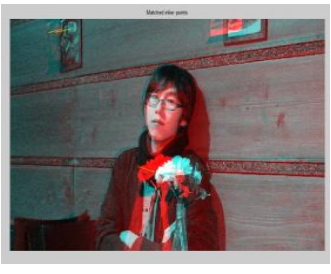

Fig 10: Matched inlier points boy

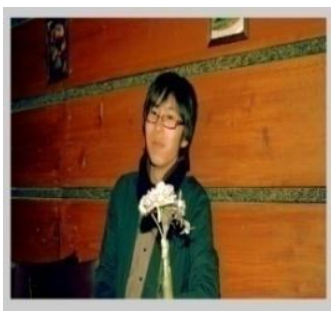

Fig 12: Enhanced image boy

\section{COMPARISONS BETWEEN SIFT AND SURF}

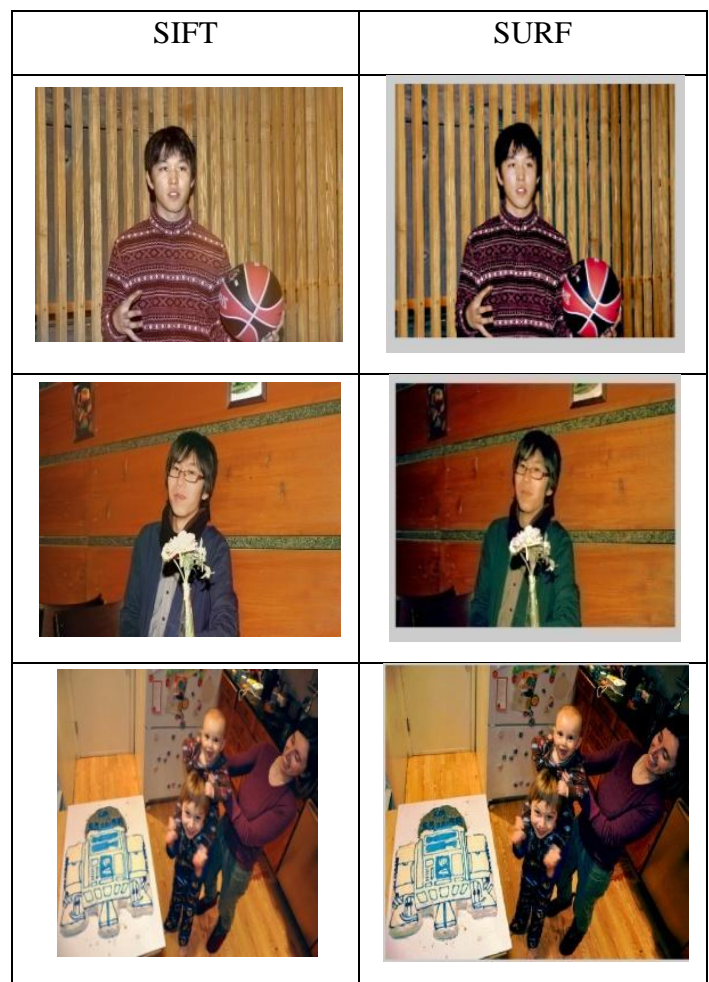

The proposed method aims for images with sharp edges and local details of the flash image and color of the long exposure image. The proposed method has higher contrast than the previous method [19] based on SIFT. The average computational time for the previous method [19] is 39 seconds where as in the proposed method average computational time is 7.4 seconds.

\section{CONCLUSION}

In this paper, proposed a new Method for Restoration of Color Images. This method restores a sharp image using flash and long exposure image pair. The resultant image obtained using proposed method has higher contrast than the previous method based on SIFT. The conventional methods for color transfer give an unnatural image and have low contrast. SURF is several times faster than SIFT. Time consumption is less when compared to SIFT based previous method. The average computational time taken by SURF based proposed method is 7.4 seconds which is lesser than average computational time taken by SIFT based previous method i.e. 39 seconds. The proposed technique can also be implemented using BRISK (Binary Robust Invariant Scalable Key points).

\section{ACKNOWLEDGEMENTS}

We would like to thank the authors of [1-19] for kindly providing their essential information and codes and would also like to thank my professors, teaching and non-teaching staff of my department for their valuable comments and suggestions which helps us to complete this work successfully. 


\section{REFERENCES}

[1] A. Buades, B. Coll, and J.-M. Morel, "A review of image denoising algorithms, with a new one," Multisc. Model. Simul., vol. 4, no.2, pp.490-530, 2005.

[2] A. Buades, B. Coll, and J.-M. Morel, "A non-local algorithm for image denoising," in Proc. IEEE Conf. CVPR, vol. 2. June. 2005, pp. 60-65.

[3] M. Elad and M. Aharon, "Image denoising via sparse and redundant representations over learned dictionaries," IEEE Trans. Image Process., vol. 15, no. 12, pp. 3736-3745, Dec. 2006.

[4] P. C. Hansen, J. G. Nagy, and D. P. O'Leary, Deblurring Images: Matrices, Spectra, and Filtering, vol. 3. Philadelphia, PA, USA:SIAM, 2006.

[5] K. Dabov, A. Foi, V. Katkovnik, and K. Egiazarian, "Image denoising by sparse 3D transform-domain collaborative filtering," IEEE Trans. Image Process., vol. 16, no. 8, pp. 2080-2095, Aug. 2007.

[6] A. Beck and M. Teboulle, "Fast gradient-based algorithms for constrained total variation image denoising and deblurring problems," IEEE Trans. Image Process., vol. 18, no. 11, pp. 2419-2434, Nov. 2009.

[7] Q. Shan, J. Jia, and A. Agarwala, "High-quality motion deblurring from a single image," ACM Trans. Graph., vol. 27, no. 3, pp. 73:1- 73:10, 2008.

[8] S. Ono and I. Yamada, "A convex regularizer for reducing color artifact in color image recovery," in Proc. IEEE Conf. CVPR, Jun. 2013, pp. 1775 1781.

[9] G. Petschnigg, R. Szeliski, M. Agrawala, M. Cohen, H. Hoppe, and K.Toyama, "Digital photography with flash and no-flash image pairs," ACM Trans. Graph., vol. 23, no. 3, pp. 664-672, Aug. 2004.
[10] T. Baba, R. Matsuoka, S. Ono, K. Shirai, and M. Okuda, "Flash/no-flash image integration using convex optimization," in Proc. IEEE ICASSP, May 2014, pp. 1185-1189.

[11] K. He, J. Sun, and X. Tang, "Guided image filtering," IEEE Trans. Pattern Anal. Mach. Intell., vol. 35, no. 6, pp. 1397-1409, Jun. 2013.

[12] K. Shirai, M. Ikehara, and M. Okamoto, "Noiseless no-flash photo creation by color transform of flash image," in Proc. IEEE ICIP, Sep. 2011, pp. 3437-3440.

[13] S. Zhuo, D. Guo, and T. Sim, "Robust flash deblurring," in Proc. IEEE Conf. CVPR, Jun. 2010, pp. 2440-2447.

[14] H.-J. Seo and P. Milanfar, "Robust flash denoising/deblurring by iterative guided filtering," EURASIP J. Adv. Sig. Process., vol. 2012(1), no. 3, pp. 1-19, 2012

[15] R. Matsuoka, T. Baba, M. Okuda, and K. Shirai, "High dynamic range image acquisition using flash image," in Proc. IEEE ICASSP, May 2013, pp. 1612-1616.

[16] R. Matsuoka, T. Yamauchi, T. Baba, and M. Okuda, "Weight optimization for multiple image integration," in Proc. IEEE ICIP, Sep.2013, pp. 795-799.

[17] S. Cho and S. Lee, "Fast motion deblurring," ACM Trans. Graph., vol. 28, no. 5, pp. 145:1145:8, 2009

[18] L. Mai and F. Liu, "Kernel fusion for better image deblurring," in Proc. IEEE Conf. CVPR, Jun. 2015, pp. 371-380.

[19] Misaligned Image Integration with Local Linear Model by Tatsuya, Ryo Matsuoka, Keiichiro Shirai and Masahiro Okuda. 